\title{
Splenic rupture: a rare presentation of metastatic choriocarcinoma
}

\author{
S. Nethra • Jed Hawe • Jackie Elder
}

Received: 26 August 2010 / Accepted: 12 September 2010/Published online: 6 October 2010

(C) Springer-Verlag 2010

Keywords Choriocarcinoma $\cdot$ Splenectomy $\cdot$ Postpartum

\section{Introduction}

VP is a 37-year-old lady who presented to the Accident and Emergency (A\&E) department with a history of left upper quadrant pain of 1-week duration. She had been seen previously by the medical team with a potential diagnosis of pulmonary embolus. On her first admission on 17 November 2009, she had a chest x-ray, ECG, D/Dimer and pulse oximetry and was given a therapeutic dose of Tinzaparin to treat for suspected pulmonary embolism. She was discharged home on 18 November 2009 after organising outpatient ventilation/perfusion (VQ) scan for the following day. Immediately following the VQ scan on 19 November 2009 which had been reported as negative, she felt unwell with shoulder tip pain, worsening left upper quadrant pain and feeling dizzy.

\section{Case presentation}

On admission to A\&E, she was pale and clammy and was found to be haemodynamically unstable with tachycardia and hypotension. She was resuscitated with intravenous fluids and further investigations arranged.

S. Nethra $(\bowtie) \cdot J$. Hawe $\cdot$ J. Elder

Countess of Chester NHS Foundation Trust,

Liverpool Road,

Chester CH2 1UL, UK

e-mail: snethra@hotmail.com
In view of the presentation, a urinary pregnancy test was performed as part of departmental protocol despite a history of a recent period, which was positive. A gynaecological opinion was sought and the serum beta sub-unit of human chorionic gonadotrophin ( $\beta \mathrm{HCG}$ ) was quantified and found to be $182,615 \mathrm{IU} / \mathrm{L}$. The chest $\mathrm{x}$-ray (CXR) performed 2 days earlier was urgently reported and showed multiple opacities in the left lobe of the lung.

She was 6 months postnatal and gave a history of irregular periods from 4 weeks postnatal, which she attributed to breastfeeding that she had continued for the first 18 weeks. In her past obstetric history, she had two normal vaginal deliveries, the last pregnancy being 6 months earlier, without complications. She was using condoms as contraception since delivery.

On examination, there were signs of generalised peritonism. A focused assessment with sonography in trauma (FAST) USS in A\&E identified free peritoneal fluid. She responded well to resuscitation allowing urgent transfer to emergency theatre and a diagnostic laparoscopy to be performed to aid diagnosis, as the very high level of HCG was not in keeping with a ruptured ectopic pregnancy but more with trophoblastic disease.

In theatre, laparoscopy revealed a haematoperitoneum of 11 , with normal fallopian tubes, uterus and ovaries, liver and appendix. There were few peritoneal defects in the pelvis, but no sign of ectopic pregnancy. Fresh blood and clots were noted around the liver and spleen. Pooling of fresh blood after suction and irrigation around the spleen raised the suspicion of a ruptured spleen due to ectopic pregnancy or metastatic choricarcinoma. Transvaginal ultrasound scan was performed in theatre to exclude uterine trophoblastic disease which identified a thin and normal endometrium. 


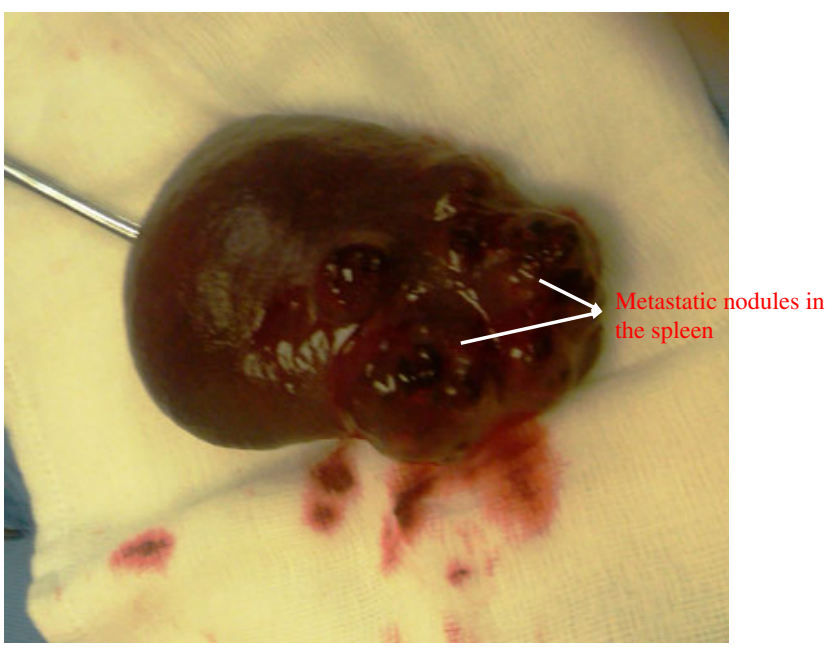

Fig. 1 Splenic choriocarcinoma

A laparotomy was performed, through an upper midline incision, with the general surgeons due to the suspected splenic rupture. After removal of clots from the splenic area, a bleeding point from the posteromedial aspect of the spleen was noted, and a decision to perform a splenectomy was made. Multiple soft nodular areas were noted on the surface of the spleen (Fig. 1).

Estimated blood loss was 1,400 $\mathrm{ml}$, and $2 \mathrm{U}$ of blood transfusion was given.

A staging CT scan of the pelvis, abdomen and chest was performed the following day. The findings were consistent with metastatic choriocarcinoma in the lungs and kidneys. No lymphadenopathy was noted and the uterus was normal. Histology of the spleen confirmed the presence of metastatic choricarcinoma with focal lymphovascular invasion. Repeat $\beta \mathrm{HCG}$ was $76,615 \mathrm{IU} / \mathrm{L}$ on day 3 post- operatively. Her blood group was recorded as $\mathrm{O}$ positive and LFTs were normal.

She was discharged home on the 5th post-operative day with follow-up in the gynaecology oncology clinic arranged after MDT discussion. Prior to discharge, adequate postsplenectomy antibiotic prophylaxis and vaccination was arranged.

After confirmation of diagnosis, she was registered with the Trophoblastic Tumour Screening and Treatment Centre in Sheffield for further management.

She had eight cycles of intravenous methotrexate, dactinomycin and etoposide. Her latest $\beta H C G$ has been $<1 \mathrm{IU} / \mathrm{L}$. She responded well, but had complete alopecia. Her follow-up programme will be serum $\beta$ HCG weekly for 6 weeks, then monthly serum $\beta$ HCG for 6 months, then 13 weekly urinary HCG for 2 years and then 6 monthly urinary HCG for life. She was advised not to get pregnant for 12 months from completion of chemotherapy.

\section{Discussion}

Splenic rupture is a very rare presentation of gestational trophoblastic disease (GTD). Mrs. VP presented with an acute abdomen and a history of irregular vaginal bleeding. High serum $\beta \mathrm{HCG}$ on admission and lung opacities on CXR all raised the suspicion of gestational trophoblastic disease. Clinical signs of shock and free fluid on the A\&E FAST scan confirmed an intraperitoneal haemorrhage and the need for immediate surgical intervention after initial resuscitation.

Gestational trophoblastic disease ranges from hydatidform mole to invasive mole to metastatic choriocarcinoma.

Table 1 GTN: FIGO staging and classification (Washington 2000), IJGO vol. 95, suppl. 1: FIGO Annual Report, vol. 26 [5]

\begin{tabular}{|c|c|c|c|c|}
\hline Stage I & \multicolumn{4}{|c|}{ Disease confined to the uterus } \\
\hline Stage II & \multicolumn{4}{|c|}{ GTN extends outside of the uterus, but is limited to the genital structures (adnexa vagina, broad ligament) } \\
\hline Stage III & \multicolumn{4}{|c|}{ GTN extends to the lungs, with or without known genital tract involvement } \\
\hline Stage IV & \multicolumn{4}{|c|}{ All other metastatic } \\
\hline \multicolumn{5}{|c|}{ Modified WHO prognostic scoring system as adapted by FIGO } \\
\hline Scores & 0 & 1 & 2 & 4 \\
\hline Age & $<40$ & $\geq 40$ & - & - \\
\hline Antecedent pregnancy & Mole & Abortion & Term & - \\
\hline Interval months from index pregnancy & $<4$ & $4-6$ & $7-12$ & $>12$ \\
\hline Pretreatment serum hCG (IU/L) & $<10^{3}$ & $10^{3}-10^{4}$ & $10^{4}-10^{5}$ & $>10^{5}$ \\
\hline Largest tumor size (including uterus) & $<3$ & $3-4 \mathrm{~cm}$ & $\geq 5 \mathrm{~cm}$ & - \\
\hline Site of metastases & Lung & Spleen, kidney & Gastrointestinal & Liver, brain \\
\hline Number of metastases & - & $1-4$ & $5-8$ & $>8$ \\
\hline Previous failed chemotherapy & - & - & Single drug & 2 or more drugs \\
\hline
\end{tabular}


Persistent gestational trophoblastic neoplasia (GTN) after live birth is very rare and the incidence is $1 / 50,000$ [1].

The primary symptoms of choriocarcinoma are gynaecological in only $50 \%$ to $60 \%$ of postpartum cases; the most common symptoms are dyspnoea, neurological symptoms, and abdominal pain. The delay between birth and presentation may exceed that of several years.

The majority of metastases affect the lung (75\%), vagina $(50 \%)$, ovaries, brain, and ureter. Rarer sites include hepatic, gastrointestinal tract, spleen and kidney. Vaginal bleeding is a common presenting symptom [2], but symptoms from metastatic disease can be the cause for initial presentation. The metastases are highly vascular leading to symptoms and signs of haemorrhage in the areas involved. Cerebral and intestinal haemorrhages are more commonly recognised presentations, though splenic bleeding has been reported rarely.

The $\beta H C G$ serves as a useful marker of disease aggressiveness and progression [3]. CT scan, MRI, and ultrasonography can be used to aid diagnosis.

Choriocarcinoma is known to be sensitive to chemotherapy (95-100\% cure rate) provided the appropriate therapy is given early in the course of the disease. A remission rate of $90-100 \%$ for patients with metastatic disease has been noted. Urgent referral of such cases to the GTD centres has to be made.

Disease assessment has to be done based on International Federation of Gynecology and Obstetrics (FIGO) staging (Table 1). High-risk women (scoring more than seven) would be given intravenous methotrexate followed by dactinomycin and etoposide 1 week later. The course is then repeated after 1 week. Treatment is continued, in all cases, until the HCG level has returned to normal and then for a further six consecutive weeks. According to the FIGO scoring system, our patient scored 7, thus, needing multi-agent chemotherapy as she is high risk with a cure rate of $95 \%$ [1].

Serial testing of serum concentrations is essential in monitoring treatment and confirming remission. Monitoring of human chorionic gonadotrophin concentrations should be continued for life as late recurrences may occur [4].

The prognosis for women with GTN after non-molar pregnancies may be worse $(21 \%$ mortality after a live birth, $6 \%$ after a non-molar miscarriage) and is mainly due to delay in diagnosis [4].
Advice about using appropriate contraception until $\beta \mathrm{HCG}$ is returned to normal is vital. Women who receive chemotherapy can have early menopause, and if they are receiving etoposide for more than 6 months, are more prone to secondary cancers like leukaemia, colon cancer, melanoma and breast cancer.

\section{Conclusion}

Irregular vaginal bleeding with an acute abdomen as in this case should raise suspicion and should be investigated to achieve a diagnosis. I would also highlight the new A\&E guidelines to perform urinary or blood HCG on all women of reproductive age group, no matter when their last period was or even if using known reliable contraception. Also of importance in this case, is the role of laparoscopy in the stabilised patient to make a diagnosis and then allow appropriate laparotomy incision if required.

Contribution to authorship The case report was written by SN with contributions from $\mathrm{JH}$, and both were directly involved in the clinical management of the case described. Dr. Elder provided expertise with the histological findings and reviewed the written report.

Details of ethics approval Ethics approval was not sought for our case report. Our patient gave full consent for her case to be presented and published in a peer-reviewed journal.

\section{References}

1. Royal College of Obstetricians and Gynaecologists (2010) RCOG Green-top Guideline no. 38, RCOG, London

2. Nugent $D$ et al (2006) Postpartum choriocarcinoma: presentation, management and survival. J Reprod Med 51(10):819-824

3. Nimje $\mathrm{K}$ et al (2006) Case reports: case of metastatic choricarcinoma, unusual metastasis. Indian J Radiol Imaging 16(4):801-803

4. Todd et al (1998) Unusual case of choriocarcinoma occurring 12 months after delivery. BMJ 316:532-534

5. GTN: FIGO staging and classification (2000), IJGGO vol. 95, suppl. 1: FIGO Annual Report, vol. 26 